\title{
Full-blooded anti-exceptionalism about logic
}

\author{
Newton C. A. da Costa and Jonas R. Becker Arenhart \\ Federal University of Santa Catarina \\ Department of Philosophy \\ Florianópolis, SC - Brazil
}

\begin{abstract}
Problems of logical theory choice are current being widely discussed in the context of anti-exceptionalist views on logic. According to those views, logic is not a special science among others, so, in particular, the methodology for theory choice should be the same in logic as for other scientific disciplines. Richard Routley advanced one such methodology which meshes well with anti-exceptionalism, and argued that it leads one to choosing one single logic, which is a kind of ultralogic. We argue that the choice for only one correct system of logic may be rejected on the basis of the methodology proposed by Routley and, furthermore, that taking anti-exceptionalism about logic seriously recommends that a pluralist view of logic should be accepted. We call this view "full-blooded anti-exceptionalism", and the resulting view on logic, lacking a proper name, "local pluralism".
\end{abstract}

Keywords: logic; anti-exceptionalism; theory choice; Richard Routley; ultralogic; pluralism.

\section{Introduction}

Disputes in logic are commonplace: given the variety of systems of logic available, it seems that one should be able to somehow choose one of those systems as correct, or at least as the best system available in the meantime; also, if it is thought that more than one of them is to be deemed correct, then reasons should be provided for that belief. Anyhow, even pluralists - those admitting that more than one system of logic may be correct, in some sense - regard it as implausible that just any system of logic whatever may do the job in any occasion. So, whatever the persuasion on the number of correct 
logics, there is an important issue involved in choosing the most appropriate logic, once an application or a goal is set.

How is that choice to be made? There is a growing tendency to think that logic is not a special science among others, that is, that it has no privileged place as an a priori science. That implies, among other things, that logic has not special right to claim that it is known by reason alone, and whose choice may be justified in a similar a priori vein, independently of anything else. Rather, a view called anti-exceptionalism about logic is gaining currency, a view according to which logic isn't special. Its theories are continuous with science; its method continuous with scientific method. Logic isn't a priori, nor are its truths analytic truths. Logical theories are revisable, and if they are revised, they are revised on the same grounds as scientific theories. (Hjortland 2017, p.632)

Of course, the most imminent precursor of the view is none other than Quine, with his claim that logic is just another part of the web of belief, one that is not immune to revision in face of recalcitrant experience. As it is known, despite this apparently liberal view on logic, Quine was not willing to give up classical logic, which, he thought, was the underlying logic that should be used to ground current science (when appropriately regimented).

Assuming the whole anti-exceptionalist package about logic requires that one provide an account for a wide range of issues, going from how logical knowledge is obtained, what is the status of such knowledge, and - a topic discussed by Routley in a way that clearly anticipates current discussion how logical theories are rationally chosen. Indeed, if logic is just another science among others, with no special justification that differs from other sciences (especially empirical sciences), there is the open possibility to revise logic. Once one thinks that logic may change in the face of evidence, and given that there may be more than one candidate for being the new system to replace the old one, then there must be something to be said about logical theory choice. In an anti-exceptionalist setting, this account of theory choice, of course, must not differ substantially from theory revision and theory choice in other fields of science, in particular, it should be similar to theory choice in empirical sciences.

As we mentioned, Routley (1980) provided for one such methodology of theory choice, and claimed that as a result of application of such method, it is more reasonable to adopt a form of ultralogic. Basically, the idea is that the methodology is just the same for logic as in other fields of science, as well as in metaphysics: it requires, in a nutshell, that we balance the virtues and vices of each option contending to be the true logic and somehow weight them. Those having lower costs and more benefits are the winners. In order to deal with the complexity of reasoning in natural language, Routley proposes that 
a kind of ultralogic should be chosen: "the best choice of logical foundations is an ultralogic, that is, an intensional inexistential logic which is ultramodal (i.e. goes far beyond the modal), is paraconsistent, and is suitably relevant" (Routley (1980) p. 97, italics in the original; see also Routley (1980a) for further articulation).

In this paper, we propose that bringing logic closer to empirical science, as the anti-exceptionalist does, may be seen as an obstacle to the ultralogic project. In fact, it may be seen as an obstacle to attempts to provide for the choice of one, and only one, true logic. Monism about logic may be threatened. We shall argue that the proximity with science adopted by the anti-exceptionalist benefits, at least in the current stage of science, a localist and pluralist approach: logic, just as any other empirical science, should deal locally with its problems, with distinct situations requiring distinct logics. Here, to consider applications relative to "distinct situations" involves even the standard application of logic to deductive inferences: that is, even when it comes to use logic in the evaluation of arguments, situations may appear that require distinct logics for the proper study of the relevant cases. In fact, the idea that there is one all-embracing system of logic that encompasses everything would be rather atypical when compared with the situation in other branches of science. The pluralist has the advantages when proximity with science is taken seriously: distinct logics are acceptable, depending on ones' goals and on ones' targets (recall, even when it comes to evaluate arguments). Even if the goal is to study valid inferences in natural language, anti-exceptionalism seems to benefit the idea that there are many logics that should do the job. We shall make this point clearer in due time.

The structure of this paper is as follows: in section 2 we sketch the model for logical choice, as advanced by Routley (1980), and more recently by Priest (2006; 2016). In section 3 we discuss two key features of the model: adequacy to the data and scope. We argue that satisfaction of these conditions by no means entails, as Routley seems to suggest, that there is one true logic, which is a kind of ultralogic. Rather, there are many difficulties with them in the light of Routley's model of theory choice. In section 4 we advance our own solution to the relation of logic to the criteria of scope and adequacy. We argue that once the similarity of logic and other scientific disciplines is properly understood, a more local, piecemeal, approach to logic is recommended, and local pluralism is a more reasonable position. We present our conclusions in section 5 . 


\section{A model for theory choice}

Let us start by getting clear as to what is at stake when one is speaking about the choice of a logical theory. It is clear that mathematically, there is a huge variety of logical systems available. As objects of mathematical study, perhaps each one deserves attention as any other, with no issue as to which is right, or which should be rationally chosen. This is a kind of pure systems pluralism. No one would deny that. Also, it is clear that distinct systems of logic may be applied in a wide array of applications, as classical propositional logic may be used in the study of electrical circuits, and some paraconsistent systems of logic may be used in the development of databases, or perhaps in software for the control of traffic lights. This may be called applied logical pluralism, and it is also fairly non-controversial.

However, it is not this kind of multiplicity of applications that is typically under discussion when it comes to logical choice (although the model of logical choice to be presented could well be applied in these cases too). Rather, it is to the so-called canonical application that philosophers shift their attention, to the case of providing for a logical theory that accounts for valid inferences and the notions related to the characterization of validity, such as truth, conditional, and meaning (among others).

Here we shall be concerned only with the problem of canonical application, so that logic should be understood as applied to the study of inferences. As Priest (2016), p.39 puts it:

The central notion of logic is validity, and its behaviour is the main concern of logical theories. Giving an account of validity requires giving accounts of other notions, such as negation and conditionals. Moreover, a decent logical theory is no mere laundry list of which inferences are valid/invalid, but also provides an explanation of these facts. An explanation is liable to bring in other concepts, such as truth and meaning. A fully-fledged logical theory is therefore an ambitious project.

It is to a study of valid inference, as it appears in natural languages and common situations involving argumentation, that logic, understood according to the canonical application, applies. The dispute concerns which system better systematizes those inferences, and which provides for a better explanation of them (we assume, with Priest (2016) p.39, that providing for a system of logic involves also specifying an intended semantics appropriate for that system, so that even if a system $\mathrm{S}_{1}$ is formally a subsystem of $\mathrm{S}_{2}$, their intended semantics may distinguish them; this is clearly the case for classical and intuitionistic logics, for instance). 
The anti-exceptionalist believes that disputes concerning this issue should be resolved as any other dispute concerning theory choice in science, and, perhaps, as we typically seem to solve practical issues concerning choice in everyday life. In fact, Routley goes as far as to put theory choice in logic in the same level as other simple kinds of choice in daily life:

Choice of a logical theory is a special case of the choice of a theory or a system, and choice of these does not differ in principle from choice of such diverse items as a new house, a winner (e.g. of a gymnastics or equestrian contest), or of a recording of a symphony. (Routley 1980, p.81)

As it is understood by the anti-exceptionalists, (at least by Routley (1980), Priest (2016) and Hjortland (2017), see also Russell (2014)), theory choice involves a kind of abductive argument, which results from the evaluation of the merits of the diverse theories on a list of desiderata that should be met by theories in the field under investigation. Empirical theories, for instance, should fare well in empirical adequacy, fruitfulness, simplicity, non-adhocness, mathematical tractability (when the theory is mathematized), and so on. For other kinds of theories or situations involving choice, other factors could have further importance.

So, while the general procedure for choice is the same in every case, what is different in different case studies are the factors that must be taken into account. Logic requires that some special factors are taken into account, factors that, for instance, would not be taken into account when choosing a record of a symphony or a new house. Routley (1980) sect. 2 lists among the important factors or criteria to be taken into account in logical choice the following ones:

i) Extensive scope: logic should have universal applicability, not only as a foundation of extensional mathematics, but working also for other very dissimilar fields such as linguistics and philosophy;

ii) Conformity to the facts: there may be some (few, perhaps) logical facts which a theory should account for; Routley provides for some instances: a conditional should count as false when its antecedent is true and its consequent is false; set inclusion is transitive (for further facts that are relevant for our discussion of Routley's views, see the text ahead). Any theory failing to account for such facts should not be taken seriously as contenders in the dispute for the correct logical theory.

iii) Accountability of the data: Routley distinguishes theory dependent data from facts. As an example of theory dependent data that must be accounted for, are the linguistic data, as well as, more controversially, Russell's 
set (the set of all sets that do not belong to themselves), which is deemed as a data of set theory. As a result, it should figure in any account of set theory; contenders failing to take it into account, such as Zermelo-Fraenkel set theory are also out of the game, while others, such as von Neumann-BernaysGödel theory, account for it as a proper class, which deems the treatment excessively artificial, and, thus, inappropriate too (according to Routley, at least).

Other criteria involved in theory choice include systematization, simplicity, fruitfulness, explanatory power, among others famous from discussions in philosophy of science (Priest (2016) also lists such virtues, while not distinguishing facts from data; the distinction is, of course, problematic, and we shall comment it further in section 4).

Once a list of criteria for theory evaluation is specified, the model proceeds as follows (here we present the model advanced by Priest (2016), given that his exposition is very similar to the one advanced by Routley, and that the details of the model are of less importance, but rather the same background idea seems to be working in each proposal; see also Priest (2006) chap.8). Suppose we are given the list of factors that must be taken into account in logical theory choice:

$$
c_{1}, c_{2}, c_{3}, \ldots c_{n}
$$

Given a logical theory $T$, we may attribute for each $c_{i}$ a score according to a scale ranging, say, in the real numbers from -10 to 10 (the choice of a range is rather arbitrary, of course), according to a measure function $m$. So, the value $T$ receives for $c_{i}$ is $m\left(c_{i}\right)$. Suppose, for instance, that a system of logic has a conjunction that fails to be true when both of its conjuncts are true. One could think that it fails badly in accounting for the facts, and, supposing that $c_{i}$ represents adequacy to the facts, would like to represent the fact that it does really bad in accounting for the facts by attributing $m\left(c_{i}\right)=-8$.

But evaluating how each system fares according to each criterion is not all. The distinct criteria $c_{i}$ may also differ on the weight or importance attributed to them. Adequacy to the facts is of utmost importance: a logic failing to account for the facts is surely not a serious contender. Simplicity, on the other hand, is a rather ambiguous criterion, which may prevent that important changes are made in the system when further evidence appears, so that it may be deemed less important, say, than explanatory power (in general, there is a balance to be achieved between keeping the system simple, and accounting for more evidence).

This difference in the relevance of each criterion is reflected in the model by attributing each criterion $\mathrm{c}_{i}$ a weight $\mathrm{w}_{i}$, which is taken into account in 
the evaluation. In the end, once every criterion receives a weight and a value according to the measure $m$, we have what Priest (2016) calls a rationality index for theories, a weighted sum of each of the criteria:

$$
p(T)=m\left(c_{1}\right) w_{1}+m\left(c_{2}\right) w_{2}+\ldots+m\left(c_{n}\right) w_{n}
$$

The theory that fares better on such a computation is to be preferred. Of course, as Priest (2016) is the first to recognize, the model is simplistic and it is not intended to capture an actual practice of theory choice. It merely attempts to capture the informal dialectic that is involved in theory choice situations. As we have mentioned, the details of the model are less relevant than the general idea that some criteria are weighted and compared, and that some are more important than others. Furthermore, the model in itself is neutral as to which should be preferred, monism or pluralism about logic (see Priest (2016) p.41). As we shall see, Routley (among others) employs it to support a form of monism, but that is due to further considerations adduced to the model, not because of the nature of the model of theory choice.

Here we shall take it for granted that this kind of approach may be seen as common to theory choice across different sciences, and even in daily life matters. We shall focus our discussion in the idea that, once logic is thought to be one only among other sciences (one of the tenets of the anti-exceptionalist thesis), the idea that scope is to be given preference loses weight, as well as the conclusion reached by Routley (and Priest, among others) that classical logic is wrong, and that a non-classical logic should be preferred. In fact, a localist pluralism will emerge as the most plausible option. Roughly put, a localist pluralist holds that distinct logics may be appropriate to deal with distinct situations, even when the application involves the study of valid inferences. The monist, of course, holds that one system of logic is correct for all situations where one is concerned with validity and inferences.

Before going ahead, let us mention that Routley (1980) sect. 4 lists further "facts" that should be accounted for. These facts are taken for granted, and provide, according to Routley, for evidence that classical logic, in particular, is ruled out as an inadequate candidate in the dispute concerning the correct logical theory:

Fact 1) Much of our discourse is intensional (while classical logic is extensional).

Fact 2) Much of philosophical discourse is about the non-existent.

Fact 3) There are inconsistent non-trivial theories and inconsistent nontrivial situations (while classical logic is explosive in the face of inconsistency).

Given that classical logic is unable to account for those facts, or at least 
not with without much distortion and trickery, leads Routley to declare that classical logic fails in providing for the basic requisite of accounting for the facts. It is as if an empirical theory provided for wrong empirical predictions. The logical system chosen by Routley to do the job is a kind of ultralogic that takes into account all the relevant features of natural language and of logic as a universal foundation for a wide range of inquiries. In fact, Routley did not develop such a logic in all its details (see Routley (1980a)), but the idea seems very close to the current logical monists, those defending that there is only one true logic that accounts for all the important facts about the consequence relation when the canonical application is taken into account (see Priest (2006) chap.12 and Read (2006) for further defenses of versions of monism).

What lies behind the demand that one system should account for those facts is the idea that logic should account for the whole variety of inferences and features of natural language (so, scope is also highly valued). We now consider the claim that as a result of those requirements, one should choose a kind of ultralogic as a universal logic.

\section{Adequacy and scope}

Monism about logic, or about the canonical application of it, at least, seems to be a pretty reasonable position on a first sight: there is one set of phenomena, argumentation and (deductive) inference in natural language, and there are facts and data about it that a logical theory should get right. There simply cannot be many correct theories about it, given that those theories disagree on which inferences are valid (see Priest (2006) chap.12 for further discussion about monism and pluralism). While it is contentious which theory has indeed got it right, for the monist there can be no discussion about the fact that there is a single correct description about consequence.

That may be a simplistic description of the underlying motivation of the monist, but we hope it is not an excessively unfair description of the idea guiding the monist enterprise. As we have seen, Routley seems to be guided by one such idea when proposing that logic should have the broadest scope as possible (and he goes on and quotes none other than Kant to support such a view! See Routley 1980 p.83). In fact, people reason under a wide array of conditions or situations, where modal notions appear, where inconsistent concepts are constantly employed (consciously or unconsciously), where situations encompassing indeterminacy of truth values are plenty, where talk about non-existent, fictitious entities is common, and so on. Those seem to be part of the data and, as Routley has put, those most varied cases furnish 
some of the data for logic.

Given the above model on logical theory choice, where adequacy to the data and to the facts is highly valued, and where scope is also valued, a system of logic accounting for all those features is to be praised as a more reasonable choice than systems that cannot account for such features. In fact, failing to account for those features is a reason for one to discard classical logic, among others, as a reasonable candidate. At least, Routley seems to believe so.

So far, no such ultralogic that is an "intensional inexistential logic which is ultramodal (i.e. goes far beyond the modal), is paraconsistent, and is suitably relevant" (Routley (1980) p. 97) was completely developed. Routley outlined the requirements and ideas behind the project, but it was not fully developed. In fact, the basic suggestion was that such a system of logic would indeed be rather weak (in order to be very general, encompassing the most diverse kinds of situations), allowing for a lot of context-sensitivity in permitting one to somehow obtain features of distinct systems in distinct situations (see Routley (1980a)). In situations that are complete and consistent, ultralogic should recover classical logic. In incomplete consistent cases, it should recover some kind of paracomplete logic (a logic violating a version of the excluded middle). In cases that involve contradictions (such as when dealing with semantic paradoxes, Cantorian set theory), a certain dialetheic paraconsistent logic should be recovered. And so on. So, the suggestion brings together the existence of only one correct system of logic, and also the idea that distinct contexts may force such logic having a definite, more narrowly defined, shape. That would be the optimal system on what concerns adequacy to the facts and the data, as well as to the scope. None other could compete with that, not even Priest's LP, it seems.

In particular, in replacing a dominant system by a new system, especially if the new system is non-classical, it is advisable that the new system preserves the sphere of application of the old system where it did succeed. Routley (1980) p.95 advises that one should be able

to provide a place (preferably a comfortable place) for what is superseded. It is good, common, logical sense - especially where so much is unknown, as is the position with nonclassical theories, in particular nonclassical theories which do not simply include the classical - to be able to allow that classical theory succeeds for a very significant class of cases (of course it succeeds where it succeeds, which is in all classical situations, and indeed for many ordinary cases (e.g. everyday finite consistent situations).

Of course, in the context of a ultralogic such strategy is generalized, so Australasian Journal of Logic (15:2) 2018 Article no. 3.6 
that inconsistent situations have their place, consistent classical situation have theirs (where classical logic holds), incomplete situations have a place too, and so on. Given the ultralogic, one should be able to plug the features of the context required and obtain the corresponding inferences, as a special case of the general logic. This would be similar to obtaining Newtonian mechanics as a special case of relativistic mechanics, when certain conditions are met.

However, what is seen as a most praised virtue of a universal system of logic (its wide scope and adequacy) is in fact a problem, which, we shall argue, makes pluralistic localism more plausible.

The first problem with this strategy of re-gaining distinct systems from a universal system, depending on the kind of context under study, is basically equivalent to the local pluralism defended, for instance, by da Costa (1997) and also by Bueno (2010). Consider the Principle of Adequacy, stated by da Costa (1997) p.55, which consists roughly in the claim that one ought to choose the most adequate logic for any given context. Here, a context is understood by da Costa as a scientific theory, but one could broaden the scope to encompass conversational situations, or fragments of dialogue where deductive reasoning is employed. What then is the difference between the local pluralist, which will choose the logic that best suits a context, from the universalist, that somehow recaptures a most adequate system inside her universal system? It seems that the difference is merely verbal, and so, both positions could handle the same cases with the same strategies. In fact, the universalist seems to be in a more delicate position of having to be able to show that the required local system is indeed a particular case of her general posited system, something that may not be possible to do, depending on the features of the universal system chosen and of the local system demanded.

But here Routley could have an answer. In fact, he is against such a "geographical" view of logic, with borders so well defined, where each system finds an application. The local pluralist seems to be worst off, it could be said, because the position ends up requiring that we should be able to separate specific situations where each system of logic would apply (this argument is also presented by Priest (2006)). However, notice that this objection, if it works, backfires on the monist: given that there are no clear borders of application of distinct systems of logic, how would it be possible to find a welldefined place, for instance, for classical logic? Well, even for the universalist the borders must be well defined, if a kind of recapture in distinct situations is to work.

The main worry behind the geographical complaint is that one may wish to reason in borderline situations. For instance, a situation involving incomplete cases (where there are, perhaps, truth value gaps), and where there are 
inconsistencies. Both kinds of cases must be taken into account. Given that the universalist will be able to account for that, the local pluralist will be able to do that too: just use the same system that the universalist, but now considered as accounting for the situation in question. That is, consider the new domain as a newly specified region to be accounted for. Geographical borders may change. Again, there are no major differences between both approaches; every time a universalist may find a system that recovers some features of a specific situation, the localist will be able to do that too.

But the positions are not equivalent on all counts. It seems the universalist monist is positing a universal background logic that must account for each specific situation, but which is idle when it comes to real application: in fact, in every situation requiring logical treatment, there are always some constraints that are holding, and the full universal logic never does any specific job. In this sense, the universal scope is illusory, and the localist has the benefits of economy. We shall also argue in the next section that the localist is in fact more fine-tuned with current scientific practice, and if we are going to embrace a legitimate anti-exceptionalism about logic, localism is preferable to the universal approach to logic as espoused by Routley and other monists.

Talk about adequacy (the most praised of the criteria) is also problematic for a universalist or monist. In fact, the idea is that one should prefer a system that is able to account for all the features of language and inferences, which, as we listed before, are widely varied. Also, recall that in order to do that, one should plug to the universal underlying logic the situations being treated (despite the geographical complaint), and recover the appropriate logical system. However, there are clear concerns on whether the kind of recovery strategy that must be employed in these cases really is able to keep faithful to the data. We shall argue that there are obstacles to the straightforward claim that a universal logic accounts for the data.

Perhaps, the most discussed case of recapture in the literature consists of the recapture of classical logic inside paraconsistent systems. For a specific case, consider paraconsistent logics such as Priest's LP, which is a candidate for the correct logic according to some. While (informally) it is able to account for inconsistent situations, it has many difficulties in expressing consistent situations. Truth is always compatible with falsity, so that when reasoning with consistent situations, where a true sentence is typically thought to be " "just true", one is at a loss. There are no simple ways to do that (to introduce a special predicate J for "just true", for instance, won't work; see Scharp (2018)). One of the strategies to recover classical logic in those contexts is Beall's shrieking rules (Beall (2013)). Basically (we are simplifying), the idea consists in adding to each consistent predicate $\mathrm{P}$ (or 
even for each theory T) a shrieked version !P which says that statements involving $\mathrm{P}$ are either consistent or trivial; this would emulate the behavior of a classical situation, incompatible with true contradictions (we follow Scharp (2018) in the notation; for details, see Beall (2013) and Scharp (2018)).

Now, although shrieked predicates seem to emulate the behavior of predicates in classical situations, the result looks really bad when it comes to judge on the adequacy to the data of the resulting system. As Scharp (2018) convincingly argues, we run the risk of misrepresenting any debate between a dialetheist and a classical logician. Adapting an example by Scharp, consider a dialetheist who claims that "it is true and false that Socrates is in the room". Now, consider a classical logician disputing that claim, who uses the predicate in a consistent manner, claiming that "it is true that Socrates is in the room (with the possibility of it being also false ruled out)". According to the shrieking strategy, the classicist is really claiming that the predicate "to be in the room", when applied to Socrates, is consistent or trivial. In the end, this is another predicate, not the one that was in the discussion to begin with. That is, after going through a shrieking process, the original predicate changes in order to include the required clause for avoiding the possibility of a true contradiction. The classical logician, however, still uses the predicate in a consistent, non-shrieked manner, so that after the shrieking process the classical logician and the dialetheist will no longer be talking about the same predicate; the contenders may be talking past each other. Worst yet: a shrieking version of every predicate of the language which may be subject of consistent discourse must be introduced if we are to account for consistent talk using those predicates. In the end, there is a complete revision of predicates of natural language. That is clearly the kind of result that one would expect from those willing to capture the facts, to correctly describe the data; indeed, it requires some reforming of natural language.

This same kind of complaint may be present in other kinds of recapture strategies. Whenever a given situation is stipulated, in order for the sentences of the universal language to be confined to that situation, a kind of recapture strategy must be employed, a strategy that will end up distorting the very sentences we began with, failing to account for the data. Nothing wrong with prescribing a reform of the vernacular, but that is not the goal of the universalist, or of the monist in general.

As a result of the discussions in this section, we achieved the following results: although universal scope and adequacy to the data are some of the points Routley puts more emphasis on, and are those criteria which seem to boost his proposal of a single ultralogic (and as a result, of the idea that a unique system must be chosen), there are problems with satisfying both of the criteria. Universal scope still requires that one treats specific 
situations piecemeal, so that the position does not distinguish itself from a local pluralism. Furthermore, local pluralism is more economic, in not positing idle universal structure acting on the background. Adequacy to the data is also difficult to grant due to the kind of distortion introduced by recapture strategies. In particular, monists seem to concede that consistent situations are part of life too, and that they must be recovered in their favorite logic. Doing that requires that some approach to obtain classical inferences be presented, and we have suggested, following Scharp (2018) that such approaches will fail to keep in touch with the initial data. Some working on the form of the sentences of natural language will have to be made, and this provides for incongruences with the original data.

\section{Going completely anti-exceptional}

But once the proposal for a universal ultralogic seems to fail in the terms of the proposed model of theory choice, what are we supposed to do? How to account for the data? What are we to make of scope? Our suggestion is that a kind of local pluralism about logic is advised if one goes completely anti-exceptionalist (or, at least, if one takes anti-exceptionalism completely seriously). Our point is that perhaps when logic (under the canonical application) is brought even closer to other sciences, then, the difficulties presented before for a universalist monist picture disappear. In fact, one may better appreciate the virtues of the kind of local pluralism that is presented here. That means that some of the constraints put by Routley on the most appropriate system of logic will have to go.

The first problem is that such a universal applicability, or universal scope, seems to be rather at odds with the piecemeal approach that characterizes current scientific methodology. While indeed unification is a virtue in science, unification does not mean that scientists dream of bringing under the same theoretical apparatus disparate fields as economics and space-time theories. Indeed, if we examine the tenets of the anti-exceptionalist, the idea that logic uses the same methodology of science, then, perhaps, the simple claim that one logic should account for all the phenomena may be seen as fragile, and so, not in tune with current scientific practice.

In fact, current practice of science is much more local and fragmented than is generally acknowledged in discussions outside philosophy of science. As Bueno (2017) p.229 clearly puts the issue, a scientific monist is one who believes that there is a single domain to which all scientific theories apply. A pluralist, on the other hand, believes that there is a plurality of scientific domains, a plurality of scientific theories, and the theories that are well 
succeeded are empirically adequate for their domains. The pluralist seems to be much better off on the account of current science:

It seems to me that any monist view faces considerable difficulties to make sense of contemporary science, where there is extremely little in common among different domains. Just consider how diverse the following theories and domains of scientific inquiry are: quantum field theory and pediatrics, molecular biology and cultural anthropology, biochemistry and sociological social psychology. They do not share goals, methods, theories, or approaches. (Bueno (2017) p.229)

Notice that science is being taken seriously in this case. Our claim will be that something similar -that is, a form of disunity or pluralism - is manifest also in the smaller scale, when we confine ourselves to the case of logic; that is, in applying logic, there is also a variety of kinds of phenomena, even if we set ourselves the task of studying deductive inferences, and distinct methods and approaches may be required in order to deal with each kind. Inferences are used for a wide variety of purposes, in very different contexts, so that distinct approaches for each of them may be studied.

Of course, one may argue that the phenomenon is only one: inference. However, even inference comes in variety of kinds that are not as uniform as usually thought. For the sake of argument, consider the distinction between inductive and deductive inferences. Both are inferences, but they require distinct approaches. For deductive logic, consider situations involving plurals: it is known that in some cases, a plural logic may be more appropriate to capture the idiosyncrasies of arguments involving pluralities, but, at the same time, such logics cannot deal with deontic reasoning. That is no reason for one to argue that a deontic plural logic is the correct one, for yet another kind of phenomenon is still left out (e.g. alethic modality). Our pluralism is not of the kind that claims that distinct logics may do the same job: rather, that the approach to reasoning, when that is the chosen application, should be performed piecemeal, taking into account the salient features of the field of application and the goals of such study. This also should make clearer what we mean by the claim that this is a "local" form of pluralism: instead of claiming that the same field of investigation may be correctly approached by distinct and incompatible systems, we claim that distinct systems may be locally more appropriate for distinct tasks. That is, the form of pluralism defended proposes that even in the case when the object of investigation is deductive argument, a pluralist approach inside logic may be favored, in the sense of a division of labor. It is this kind of approach, we claim, that seems much closer to scientific practice in other fields. 
To favor this view, we begin by pointing to the claim that natural language, by itself, does not seem to have a logic of its own. In fact, our claim is that language is used in many distinct ways, even when argumentation is the target, in order for us to select it all as a field of study. As Smith (2011) and Glanzberg (2015) among others have already pointed out, it is difficult to see natural language as involving a logic, given that logic requires idealization and, to some degree, sharpening and perhaps even distortion of some features of the target object of study.

This brings us to an important analogy between science and logic that is generally not taken into account, even by anti-exceptionalists, and which will provide for further evidence that the kind of pluralism we have been advancing is a more promising candidate for a correct understanding of logic, at least as it is practiced today. To begin with, logic is a mathematical science. The mathematical aspects of logic are just as well-developed and just as abstract as any other branch of mathematics (this is discussed in da Costa (1997)). As we have already discussed, the canonical application of logic to deductive inference may be considered as privileged over other applications, such as electrical circuits, but it is an application, nonetheless. In this sense, discussions of applying logic to inference may benefit from discussions of applications of mathematics to natural phenomena in general.

We do not have space to discuss all the relevant features of applying mathematics, and logic in particular, to the study of inference, but we shall confine ourselves to the one we think will make clearer our claim that natural language does not have a logic of its own and that the pluralist position we advanced is the most appropriate. It concerns the role of idealization in science (again, if we are going to take seriously the anti-exceptionalist claim that logic is continuous with science, these aspects should bear some similarity). This will also help us deal with the problem of scope and adequacy to the data, questions we raised in the beginning of this section.

What is the role of idealization in logic? Idealization is involved in at least two related stages of applying logic: the selection of the data and stipulation of the target phenomena, and the development of the mathematical systems that will account for the data and the phenomena.

In the first case, viz. selecting the phenomena, idealization helps us preparing the data, just as it helps physicists in setting their experiments and reading the results of those experiments. In the case of logic, as Smith (2011) has pointed out, one cannot simply select inference or consequence and then go ahead to apply a mathematical structure to describe it. Rather, one puts some filters that somehow sharpen the phenomena into workable units and provides for conditions of the application of mathematical theory. There are important questions to be answered once inference is settled as the 
field of application, and before the mathematical apparatus may be applied or developed. There is no raw notion of inference waiting to be captured. To illustrate the point, both of how one gets at a loss by thinking that there is one pre-theoretic notion of inference to be captured, and how the sharpening process may be developed, determining features of a more mathematically tractable notion of consequence, it is interesting to quote Smith (2011) p.29 (here, he is challenging those who believe that there is a well-defined pretheoretical notion of logical consequence):

If you think that there is, start asking yourself questions like this. Is the intuitive notion of consequence constrained by considerations of relevance? - do ex falso quodlibet inferences commit a fallacy of relevance? When can you suppress necessarily true premisses and still have an inference which is intuitively valid? What about the inference 'The cup contains some water; so it contains some $\mathrm{H}_{2} \mathrm{O}$ molecules'? That necessarily preserves truth (on Kripkean assumptions): but is it valid in the intuitive sense? - if not, just why not?

Of course, answering those questions provides for features of a consequence relation that applies in some situations (classical situations, dealing with classical mathematics, for instance), and answering them differently provides for relations that apply in other situations. It all depends on the purpose of the investigation: investigate the consequence relation that seems to be in the underlying logic of classical mathematics, or in contexts where relevance is important, or in contexts where there may be undefined truth values? The data provided by answering those questions sharpen the informal idea of consequence relation, idealize it, but also permits one to introduce the mathematical machinery of logic. Without this, as Smith (2011) argued, no mathematical theory is able to attempt to capture an informal notion. That accounts clearly for the scope of a system: the scope involves the field that is delimited in the first place.

Once this is settled, questions about the adequacy of a system to the facts and to the data get easier to answer. In fact, one could even go on and say that only after such a round of idealization it is possible to speak of facts to be described and data. Given a clear (informal) notion of consequence, one may check which system of logic better captures the relevant features of the informal idea. Notice that this is completely in agreement with the kind of pluralism we have provided for. Given that the notion of consequence may be specified in distinct directions, for distinct purposes, involving distinct relevant choices of logical vocabulary, there may be distinct sets of data once this first stage is performed, and distinct systems may do better on each. 
The second aspect where idealization gets in is in the development of the mathematical system itself. As Colyvan (2013) has argued, logic involves idealizations for many distinct purposes, just as scientific theories and models do. According to the taxonomy advanced by Colyvan (2013), some idealizations are specific for mathematical tractability, such as perhaps the truth-table of the material conditional, or the assumption that the truth assignment function is, indeed, a function (it could well be a relation). Some are due to requirements of the field of application, once one is stipulated. So, for instance, one could argue that a negation sign that explodes in the face of a contradiction does not account for the data once an inconsistent domain is chosen, or once one chooses a domain involving explicit assumption of some contradictions. In this sense, there are some data that get fixed in the first stage, as we commented, when the sharpening of the informal concept provided for, and which the system to be developed must account for. Colyvan also separates for a third kind of idealization, which is called "demands of reason". Demands of reason are closer to what Routley called facts, conditions such as the truth of a conjunction whose conjuncts are also true, or the falsity of a conditional whose antecedent is true and whose consequent is false. This category, of course, is the most debatable one, but it seems that it would account for a distinction between basic logical facts, some that cannot be absent of any logical theory willing to be applied to argumentation, and those that are more flexible, which fall in the second category of idealization and may fail in some contexts, but hold in others.

Our main contention is that the specific classification of the kinds of idealization by Colyvan is secondary to the claim that providing for a logical system to deal with a set of phenomena involves idealization in the mathematical apparatus of the logic. This brings logic closer to science too, given that mathematical idealizations are present in other branches of science as well. To demand that a mathematical description account for a wide variety of disparate phenomena makes for mathematical treatment more difficult and, perhaps impossible. One must abstract some of the features of the target domain and idealize less important features. As we have provided for an instance, when dealing with mathematical reasoning, in classical mathematics, at least, considerations of tense, of the possibility of inconsistency, among others, may be left out of the account, so that the system gets simpler. These are common features that are involved in scientific model-building, and providing for a system of logic may be seen as similar as providing for a mathematical model of a situation that is given.

Once the target situation and the model are given, one is able to judge on the adequacy of the model and provide for a kind of bootstrapping process where the model is judged against the data, and the data are judged against 
the explanatory power of the model. This accounts for the adequacy criteria, of course, and is not a matter of all or nothing, but of balancing pros and cons of the model. In fact, the very idea of adequacy may end up being revised in the light of philosophical discussion of other sciences. There is a huge literature in the philosophy of science on how we may learn from models we know are not completely realistic, but we shall not develop that discussion here. That is a clearly underdeveloped issue in logic, but one that must be taken into account by anti-exceptionalists, an issue we believe will also help us better understand the nature of logic and its relation to its applications.

\section{Conclusion}

We have presented a model for theory choice in logic that was advanced by Routley and located it in current debates on anti-exceptionalism about logic. By judging the ultralogical proposal of Routley - and implicitly along with it, typical monist tenets - in the lights of the demands of antiexceptionalism, we have argued that a universalist proposal is not preferred over a pluralistic approach. Indeed, we have advanced a story according to which one may bring logic even closer to other fields of science by taking into account idealization, abstraction, and a kind of domain sensitivity. According to that story, logic is even closer to other branches of science than is typically assumed, and there is a whole field still to be explored, on taking logical theories as models for specific kinds of phenomena and all the implications such approach may have (this kind of investigation is put forward also in Shapiro (2006)). We have called our approach "full blooded anti-exceptionalism" in order to emphasize that the similarity of logic with science is brought to other dimensions that are not (so far) typically appreciated by other anti-exceptionalists. 


\section{References}

Beall, J. C. (2013) Shrieking against gluts: a solution to the 'Just True' problem. Analysis 73, pp. 438-445.

Bueno, O. (2010) Is logic a priori? The Harvard Review of Philosophy XVII, pp.105-117.

Colyvan, M (2013) Idealisations in normative models. Synthese 190, pp.1337-1350.

da Costa, N. C. A. (1997) Logiques Classiques et Non Classiques. Essai sur les fondements de la logique. Paris: Masson.

Glanzberg, M. (2015) Logical consequence and natural language. In: C. R. Carret, O. T. Hjortland (eds.) The foundations of logical consequence, pp. 71-120. Oxford: Oxford Un. Press.

Hjortland, O. T. (2017) Anti-exceptionalism about logic. Philosophical studies 174, pp.631-658.

Priest, G. (2006) Doubt truth to be a liar. Oxford: Oxford Un Press.

Priest, G. (2016) Logical disputes and the a priori. Princípios v.23, n.40, pp.29-57.

Read, S. (2006) Monism: The one true logic. In: D. Devidi, T. Kenyon (eds.) A logical approach to philosophy: essays in honor of Graham Solomon, pp.193-209. Springer.

Routley, R. (1980) The choice of logical foundations: Non-classical choices and the ultralogical choice. Studia Logica 39(1), p.77-98.

Routley, R. (1980a) Ultralogic as universal? In: R. Routley, Exploring Meinong's jungle and beyond. An investigation of noneism and the theory of items. Canberra: Departmental Monograph, Philosophy Department, Research School of Social Sciences, Australian National University.

Russell, G. (2014) Metaphysical analyticity and the epistemology of logic. Philosophical studies 171(1), pp. 161-175.

Scharp, K. (2018) Shrieking in the face of vengeance, Analysis, anx163, https://doi.org/10.1093/analys/anx163

Shapiro, S. (2006) Vagueness in context. Oxford: Clarendon Press.

Smith, P. (2011) Squeezing arguments. Analysis 71, pp. 22-30. 